\title{
Migraine Detection From Eeg Signals Using Tunable Q-Factor Wavelet Transform and Ensemble Learning Techniques
}

Zülfikar ASLAN ( $\square$ zulfikaraslan27@gmail.com )

Gaziantep University: Gaziantep Universitesi https://orcid.org/0000-0002-2706-5715

\section{Research Article}

Keywords: eeg, migraine detection, tqwt, ensemble classifiers, kruskal wallis

Posted Date: May 19th, 2021

DOI: https://doi.org/10.21203/rs.3.rs-491719/v1

License: @ (1) This work is licensed under a Creative Commons Attribution 4.0 International License. Read Full License

Version of Record: A version of this preprint was published at Physical and Engineering Sciences in Medicine on September 10th, 2021. See the published version at https://doi.org/10.1007/s13246-021-01055-6. 


\section{Abstract}

Migraine is one of the major neurovascular diseases that recur, can persist for a long time, cripple or weaken the brain. This study uses electroencephalogram (EEG) signals for the diagnosis of migraine, and a computer-aided diagnosis system is presented to support expert opinion. A Tunable Q-Factor Wavelet Transform (TQWT) based method is proposed for the analysis of the oscillatory structure of EEG signals. With TQWT, EEG signals are decomposed into sub bands. Then, the features are statistically calculated from these bands. The success of the obtained features in distinguishing between migraine patients and healthy control (HC) subjects was performed using the Kruskal Wallis test. Feature values obtained from each sub band were classified using well-known ensemble learning techniques and their classification performances were tested. Among the evaluated classifiers, the highest classification performance was achieved as $89.6 \%$ by using the Rotation Forest algorithm with the features obtained with Sub band 2. These results reveal the potential of the study as a tool that will support expert opinion in the diagnosis of migraine.

\section{Introduction}

Migraine is one of the major neurological disorders affecting one in seven people worldwide. It manifests itself with symptoms such as severe pain in one or both sides of the brain and sensitivity to light [1]. It is reported that migraine disease ranks first among neurological disorders worldwide [2]. Presenting symptoms of migraine disease in different diseases such as tension headache, epilepsy and stroke may cause misdiagnosis. Recently, many studies aimed at diagnosing migraine with high classification achievements have been exhibited. In the relevant literature studies, it was aimed to distinguish between $\mathrm{HC}$ subjects and migraine patients by using machine learning algorithms.

The diagnosis of migraine is usually evaluated by a specialist, taking into account the patient's medical history and symptoms of the disease. First, the patient's headache history (characteristics of headache, its effects on daily life and family headache history) is analyzed. Apart from this, comprehensive examinations are made in order to make a full evaluation [3]. Techniques such as magnetic resonance imaging (MRI), computed tomography (CT) and EEG are used to support expert opinion in the detection of the disease. However, in the detection of migraine disease with computer-aided diagnosis (CAT) systems, the use of EEG is preferred because of its less cost and less equipment need among these techniques.

EEG produces non-periodic and non-stationary time-series data of electrical changes occurring between neurons in the brain [4]. There are many studies that use EEG signals, especially in the diagnosis of neurological disorders. Features obtained by different feature extraction methods applied to EEG signals are classified with a machine learning algorithm. In this context, there are studies in the relevant literature that diagnose migraine using EEG signals and machine learning algorithms.

In one study, Yin et al. [5] presented a clinical decision support system in the diagnosis of migraine. They aimed to diagnose tension headache and possible migraine in their studies. They used the detections made by the experts as a feature and classified them with the nearest neighbor (KNN) algorithm and achieved $90 \%$ success. Krawczyk et al. [6] have presented another clinical decision support system. They tested different machine learning algorithms and methods for headache classification. They achieved the highest performance at $81 \%$. Akben et al. [7] analyzed the EEG signals under flash stimulation to detect migraine disease. They obtained $85 \%$ accuracy by classifying the power spectrum values they obtained under $2 \mathrm{~Hz}, 4 \mathrm{~Hz}$ and $6 \mathrm{~Hz}$ flash excitation frequency with artificial neural network. Subasi et al. [8] investigated the detection of migraine disease and the effect of photic stimulation. First, they applied discrete wavelet transform for feature extraction from control EEG signals. Afterwards, they tested different machine learning algorithms and methods using these features. As a result, they distinguished healthy individuals and migraine patients with the Random Forest algorithm with the highest accuracy of $85.95 \%$. Akben et al. [9] aimed to find the best flash stimulation frequency for the detection of migraine in another study. They classified the power spectrum features they obtained from EEG signals for different flash stimulation frequency values with ANN. They detected migraine disease with $83.3 \%$ accuracy. In addition to the advantages of the studies mentioned in the literature in the diagnosis of migraine, improving its performance is an important need.

Recently, an tunable Q-factor wavelet transform (TQWT) is gaining popularity in the analysis of brain signals [10]-[12]. The main reason for this is that it is a flexible and fully discrete wavelet transform, which is particularly suitable for analyzing oscillatory signals [13]-[16]. Traditional wavelet transforms cannot adjust Q factors. TQWT can adjust the Q factor and be used as a powerful method for oscillating signal analysis.

In the relevant literature studies, direct feature extraction from signals, filtering methods, power spectrum analysis, FFT and wavelet transform methods are used. However, these methods have some disadvantages. Filtering and power spectrum analysis methods require selection of filter boundaries. FFT has a localization problem. Wavelet transformations, on the other hand, require the selection of appropriate decomposition levels and selection of the main wavelet. For these reasons, the TQWT method, which does not need to select the wavelet function, is used to obtain independent components due to the nature of the EEG signal [12], [17], [18]. 
With this motivation, a CAT system using the TQWT method was developed in order to distinguish migraine patients and healthy individuals with high performance. Compared to the relevant literature studies we know, the TQWT method is used for the first time in the detection of migraine disease. In this study, a newly presented EEG dataset for migraine patients and HC subjects is used. EEG data were divided into sub bands by applying TQWT after noise cleaning with Multiscale Principal Component (MSPCA) first. Afterwards, feature extraction was made on each sub band and the obtained method was tested by classifying the obtained feature values with different ensemble learning algorithms. With our proposed method, the highest classification performance was achieved with Rotation Forest with an accuracy of $89.6 \%$. The classification performance we have obtained offers a satisfactory accuracy value according to the literature studies we know.

The main contributions of our proposed method are summarized as follows:

1. We offer an automatic classification model that efficiently combines TQWT and Ensemble learning algorithms for the diagnosis of Migraine disease from EEG signals.

2. Investigation of the differences between migraine patients and healthy individuals using TQWT (through sub-bands) method.

3. Selecting the appropriate features using statistical analysis method and discovering a sustainable classifier for the diagnosis of Migraine.

4. Testing the method with different Ensemble learning algorithms to determine the best classification performance.

5. Improving classification performance compared to existing methods.

\section{Methodology}

This section presents the data set descriptions and preprocessing procedures to be analyzed first. The methodology plan suggested in the section is then presented. Figure 1 provides an overview of the proposed method for diagnosing Migraine using EEG signals.

Our proposed method includes the following stages:

i. Noise removal from EEG signals;

ii. separation of EEG signals into sub bands by TQWT method;

iii. reducing the size of TQWT sub bands by calculating their statistical features;

iv. checking the ability to distinguish these calculated features by the KW test;

v. testing the classification performance of the proposed method with different ensemble learning classifiers.

\section{Dataset}

The data set used in this study is provided by Carnegie Mellon University. The EEG record includes data on 18 adult migraine patients (12 women and 5 men, average age 27.6, age range 19-54) and $21 \mathrm{HC}$ subjects without headaches (12 women and 9 men, average age 27.9, age range 19-54). 12 people with migraine are classified as migraine with aura and 6 as migraine without aura in accordance with the criteria of the International Headache Association. EEG recordings were recorded at rest with a 128-channel BioSemiActiveTwo system using a 24-bit A/D converter with a sampling frequency of $512 \mathrm{~Hz}$. At rest, the recording time is about 6 minutes. During resting state recording, participants were asked to keep their eyes open and fix them on the black middle cross. [19].

\section{The Multiscale Principal Component Analysis (MSPCA)}

Multiscale Principal Component (MSPCA) is a combination of the features of Principal Component Analysis (PCA) and wavelet analysis. The attractive properties of wavelet analysis and Principal Component Analysis (PCA) are combined in MSPCA. Wavelet analysis is used to extract deterministic features. PCA is also applied to properties in order to determine a linear relationship between properties. MSPCA is used as a suitable tool for analysis of changing signals and noise removal due to its multi-scale feature. Allows efficient filtering of MSPCA residues and scores [20]. For these reasons, the MSPCA method was used in our study to clear the noise of EEG signals.

\section{Tunable Q-Factor Wavelet Transform (TQWT)}

Tunable Q-Factor Wavelet Transform (TQWT) can be used as an effective tool for oscillatory signal analysis [21]. Q, $r$ and $j$ are adjustable parameters. The $Q$ parameter indicates the $Q$ factor, the $r$ parameter the high-speed sampling rate, and the $j$ parameter the discrimination level. The Q parameter is used to control the number of wavelet oscillations. Unwanted excessive oscillation values on the signal are controlled by r. Basic filter sets in the TQWT structure can be specified in the frequency domain. These filters are non-rational transfer functions and can be used effectively in time-frequency analysis [22].

Page $3 / 11$ 
Wavelet transforms should have a low Q factor for signals with no or very little oscillation, while a high Q factor value is required in the analysis of oscillating signals. The TQWT method allows this Q value to be adjusted parametrically. Besides, TQWT is widely used in the analysis of various physiological signals [16], [18], [23]. Filters are computationally successful due to their use of rational transfer functions. This allows obtaining the direct representation of the signal in the frequency domain. TQWT is superior to other methods due to these advantages.

The TQWT is designed because it has limited ability to adjust Q factor in general wavelet transformations (except for Continuous Wavelet transformation). TQWT has true scaling factors and reconstruction oversampled filters. The $Q$ factor affects the oscillation behavior of wavelets and the extent to which the wavelet oscillations are maintained. The TQWT redundancy is obtained by computing the r parameter in an infinite number of levels. It is recommended to have a value of 3 or greater for the R parameter. Parameter $\mathrm{J}$ specifies the number of wavelet transform levels and filter banks. The conversion consists of a series of filter banks. As a result of the decomposition into sub bands, $\mathrm{J}+1$ sub band will be obtained [21]. In this study, the best performance was determined as $Q=1, r=2, J=8$ by testing various values of $Q, r$ and $\mathrm{J}$, and a total of 9 sub bands were obtained from each EEG channel.

\section{Feature Extraction from Sub bands and Evaluation of Features with Kruskal Wallis Test}

In this section, the size of the sub bands obtained by TQWT is reduced by calculating the statistical features. Various linear or nonlinear features are inferred from sub bands obtained by applying TQWT to the EEG signal. A total of twenty features were extracted from the lower bands. These features are activity, mobility, complexity, shannon entropy, log energy entropy, mean, maximum, minimum, standard deviation, skewness, kurtosis, median, variance, first quarter (Q1), second quarter (Q2), third quarter (Q3), fourth quarter (Q4), interquartile range, root mean square, trimmean (mean excluding outliers).

$\mathrm{KW}$ test was used in order to test the discrimination ability of the studied features. $\mathrm{KW}$ is a nonparametric method used to determine whether test samples come from separate distribution. Used to compare two or more independent samples with different or equal sample sizes. KW test was applied for all features values and it was determined that all features are meaningful features. Table 1 shows the chi $(p)$ value obtained when the KW test is applied to the activity, mobility, complexity, minima, standard deviation feature values.

\section{Classification of Migraine and Healthy Control}

In this part, it is aimed to test the classification performance of the proposed method with different ensemble learning classifiers. For this purpose, Rotation Forest, BFTree, Random Forest, Bagging, AdaBoost, SPAARC, MultiBoost, Random Tree and NBTree ensemble classifiers were used. The reason for choosing these ensemble classification algorithms is that they are widely used in the field of biomedical signal processing. The main reason for this is that these classifiers exhibit both fast and good classification performance. With this motivation, the accuracy of the proposed method was tested using the classifiers mentioned in our study. With the proposed method, the highest classification performance was obtained with the Rotation Forest classifier.

\section{Performance Evaluation Metrics}

The performance of the method proposed in this study was tested using six parameters: TPRate, FPRate, Precision, Recall, F-Measure and ROC area. The performance of a classifier is evaluated considering the error rate. A performance is calculated if the classifier correctly predicts the class of each sample, otherwise the error is calculated. Cross validation is a method that is used effectively especially in limited data situations. Our proposed method has been tested with different ensemble learning techniques. Each classification process has been evaluated by taking the cross validation value to be ten.

The values used to calculate the parameters we use to test the classification performance are: number of true negatives (TN), true positives (TP), false positives (FP), and false negatives (FN). Using these values, evaluation metrics can be calculated as shown in the formulas below.

\footnotetext{
Sensitivity $($ Recall $)=\frac{T P}{T P+F N} \times 100$

Sensitivity $($ Recall $)=\frac{T P}{T P+F N} \times 100$

Precision $=\frac{T P}{T P+F P} \times 100$

F1Score $=2 * \frac{\text { precision }- \text { recall }}{\text { precision }+ \text { recall }} \times 100$
} 
The area under the ROC curve is an important evaluation parameter used to evaluate the performance of the classifier. The higher the ROC area value, the more successful the classifiers are in predicting 0 's as 0 's and 1's as 1 . In other words, the high ROC value indicates that the success of differentiating the diseased individual from the $\mathrm{HC}$ is high [24].

\section{Results}

\section{Experimental Setup}

The data set used in this study includes EEG records of migraine patients and $\mathrm{HC}$ subjects, which can be accessed as open source. As mentioned earlier, this data set contains 128-channel EEG data of 39 individuals, 18 of whom were migraine patients and 21 of whom were $\mathrm{HC}$ subjects. There are small differences in the duration of EEG recordings between individuals. Therefore, the length of each EEG channel is taken as a vector of 195,000 lengths. MSPCA method has been applied to clean the noises in the EEG data. Afterwards, TQWT (with Q $=1, r=$ 3 and $j=8$ ) method was applied to each channel of the EEG data of each individual. A total of 9 sub band values were obtained from each channel. After that, by applying statistical feature extraction methods to EEG channels, 20 feature values were obtained from each EEG channel. For each sub band, a matrix value of (4492 instances X 20 features) was obtained, including migraine patients (18 migraines patient's $\times 128$ channels $\times 20$ features) and HC subjects ( $21 \mathrm{HC}$ subject's X 128 channels $\times 20$ features). Each sub band was evaluated separately. Experiments were carried out using Matlab software.

\section{Statistical Analysis}

The KW test was used to test the distinguishing ability of the feature values we obtained in our study. Statistical analyzes were carried out using Matlab software. The chi $(\rho)$ value $(\rho<0.05)$ in the KW test indicates the success of the discrimination performance. KW test was applied for all feature values we obtained in our study and it was observed that all features have high discrimination. The chi $(\rho)$ values obtained from the KW test for some feature values (activity, mobility, complexity, minima, standard deviation) obtained from the sub bands are shown in Table 1.

Table 1

Chi(p) value obtained as a result of the KW test of some features.

\begin{tabular}{|c|c|c|c|c|c|}
\hline $\begin{array}{l}\text { Sub Bands/ } \\
\text { Features }\end{array}$ & Activity & Mobility & Complexity & Minima & Standard Deviation \\
\hline SB1 & $1.209 * 10^{-51}$ & $4.7398 * 10^{-10}$ & 0.0150 & $1.0103 * 10^{-21}$ & $1.2127 * 10^{-51}$ \\
\hline SB2 & $9.2709 * 10^{-77}$ & $2.3155^{\star} 10^{-27}$ & $2.5122 * 10^{-56}$ & $4.3941 * 10^{-30}$ & $9.2692 * 10^{-77}$ \\
\hline SB3 & $3.7730 * 10^{-55}$ & $3.5544 * 10^{-84}$ & $1.4828 * 10^{-60}$ & $6.8961 * 10^{-27}$ & $3.7782 * 10^{-55}$ \\
\hline SB4 & $9.0269 * 10^{-15}$ & $1.5939 * 10^{-29}$ & $1.0686 * 10^{-05}$ & $4.5387 * 10^{-06}$ & $9.0248 * 10^{-15}$ \\
\hline SB5 & $5.5877 * 10^{-11}$ & $8.1770 * 10^{-07}$ & 0.0010 & $5.7394 * 10^{-05}$ & $5.5837 * 10^{-11}$ \\
\hline SB6 & $2.4151 * 10^{-12}$ & 0.0061 & $3.5577 * 10^{-04}$ & $9.1634^{\star} 10^{-08}$ & $2.4138 * 10^{-12}$ \\
\hline SB7 & $7.0895 * 10^{-14}$ & 0.0102 & $1.4758 * 10^{-04}$ & $1.0338 * 10^{-08}$ & $7.0815^{\star} 10^{-14}$ \\
\hline SB8 & $3.5733 * 10^{-12}$ & $2.3693^{*} 10^{-07}$ & $2.1212^{*} 10^{-18}$ & $4.3779 * 10^{-07}$ & $3.5740 * 10^{-12}$ \\
\hline SB9 & 0.0802 & $4.5773 * 10^{-22}$ & $2.0745^{\star} 10^{-24}$ & 0.1387 & 0.0802 \\
\hline
\end{tabular}

\section{Evaluation of Classification Performances}

In Table 2, the F-Measure values obtained by different ensemble classification algorithms of the features obtained from the sub bands are shown. The performance values of the proposed method obtained by different ensemble classification algorithms are close to each other. Classification process was carried out by taking the cross validation value of 10 . However, as seen in Table 2 , the classification performance decreases as the depth of the sub bands increases. The best classification performance was obtained with the Rotation Forest classifier for the lower band 2 value. The classification performance (F-measure value) for Sub band 2 with the Rotation Forest classifier was $89.6 \%$. Figure 2 shows the classification performances of the sub bands obtained with different ensemble classifiers. 
Table 2

F-Measure value obtained by classifying the sub bands with different ensemble learning

\begin{tabular}{|llllllllll|}
\hline & SB1 & SB2 & SB3 & SB4 & SB5 & SB6 & SB7 & SB8 & SB9 \\
\hline BFTree & 0,834 & 0,854 & 0,788 & 0,743 & 0,748 & 0,756 & 0,728 & 0,732 & 0,757 \\
\hline RotationForest & 0,882 & $\mathbf{0 , 8 9 6}$ & 0,862 & 0,836 & 0,818 & 0,826 & 0,805 & 0,792 & 0,821 \\
\hline Bagging & 0,862 & 0,878 & 0,843 & 0,807 & 0,791 & 0,796 & 0,785 & 0,779 & 0,799 \\
\hline AdaBoostM1 & 0,855 & 0,867 & 0,825 & 0,798 & 0,77 & 0,772 & 0,767 & 0,762 & 0,785 \\
\hline Random Forest & 0,888 & $\mathbf{0 , 8 9 5}$ & 0,864 & 0,831 & 0,816 & 0,824 & 0,807 & 0,799 & 0,818 \\
\hline SPAARC & 0,836 & 0,858 & 0,818 & 0,775 & 0,773 & 0,765 & 0,743 & 0,747 & 0,778 \\
\hline MultiBoostAB & 0,864 & 0,878 & 0,842 & 0,809 & 0,785 & 0,789 & 0,778 & 0,783 & 0,797 \\
\hline RandomTree & 0,821 & 0,843 & 0,792 & 0,754 & 0,739 & 0,735 & 0,729 & 0,718 & 0,746 \\
\hline NB Tree & 0,808 & 0,849 & 0,797 & 0,716 & 0,715 & 0,748 & 0,7 & 0,685 & 0,732 \\
\hline
\end{tabular}

Table 3

Evaluation metrics obtained by classifying Sub band 2 with different ensemble learning algorithms.

\begin{tabular}{|lllllll|}
\hline & TP Rate & FP Rate & Precision & Recall & F-Measure & ROC Area \\
\hline BFTree & 0,855 & 0,154 & 0,857 & 0,855 & 0,854 & 0,859 \\
\hline RotationForest & $\mathbf{0 , 8 9 6}$ & $\mathbf{0 , 1 1 1}$ & $\mathbf{0 , 8 9 8}$ & $\mathbf{0 , 8 9 6}$ & $\mathbf{0 , 8 9 6}$ & $\mathbf{0 , 9 5 5}$ \\
\hline Bagging & 0,879 & 0,129 & 0,881 & 0,879 & 0,878 & 0,941 \\
AdaBoostM1 & 0,867 & 0,137 & 0,867 & 0,867 & 0,867 & 0,932 \\
\hline Random Forest & 0,896 & 0,112 & 0,898 & 0,896 & 0,895 & 0,957 \\
\hline SPAARC & 0,858 & 0,146 & 0,858 & 0,858 & 0,858 & 0,891 \\
\hline MultiBoostAB & 0,878 & 0,128 & 0,879 & 0,878 & 0,878 & 0,94 \\
\hline RandomTree & 0,843 & 0,159 & 0,843 & 0,843 & 0,843 & 0,842 \\
\hline NB Tree & 0,85 & 0,158 & 0,851 & 0,85 & 0,849 & 0,889 \\
\hline
\end{tabular}

Table 3 shows the evaluation metrics obtained by classifying the features obtained from Sub band 2 with different ensemble classifiers. The highest classification performance has been achieved with the Rotation Forest classifier for all evaluation metrics. As a result, it reveals that the Rotation Forest classifier is the best model for our proposed method. Among the sub bands, the second highest classification performance for Sub band 1 was $88.2 \%$, while the lowest result was obtained with Sub band 9 as $82.1 \%$.

In order to better evaluate the performance of the proposed method, we draw the ROC curve obtained by classifying Sub band 2 with different ensemble algorithms in Fig. 4. The area under the ROC curve (AUC) is an evaluation metric used to measure the performance of the classifier. As can be understood in Fig. 4, the classification performance of the proposed method is obtained with the highest Rotation Forest and Random Forest algorithms. Its true positive rate was obtained as $89.6 \%$ by Rotation Forest and Random Forest algorithms. However, the false positive rate was calculated as $11.1 \%$ for Rotation Forest and $11.2 \%$ for Random Forest, respectively. This result shows that the proposed method has a high classification performance. In order to reveal that the migraine patient and HC are successfully distinguished with the proposed method, we give the Confusion Matrix value obtained by classifying the Sub band 2 with the Rotation Forest algorithm in Fig. 5. The values given in Fig. 5 show the average values obtained by taking the cross validation 10 . In Fig. 5 , it is seen that the classification success of the proposed method is $94 \%$ for $\mathrm{HC}$ subjects and $84.4 \%$ for migraine patients.

\section{Discussion}

\section{Comparison Analysis of Our Proposed Method with Existing Methods}


In Table 4, a comparative analysis report is presented with the method suggested by the relevant literature studies using different data sets for the diagnosis of migraine. In the relevant literature, the known studies based on classification in the diagnosis of migraine have been discussed in two different categories. The studies in the first category are based on the feature values obtained by expert interviews with the patient [5], [6], [25]. Yin et al. [5] applied an independent component analysis (ICA) to select the feature values they obtained in their studies. Afterwards, they were classified with KNN and achieved a classification success of 90\%. Krawczyk et al. [6] applied ReliefF Greedy for feature selection process in their study and they obtained $81 \%$ accuracy by classifying the results with Random Forest algorithm. In another study, Jackowski et al. [25] used the Backward Feature Elimination method for the feature selection process, and by classifying the features they obtained with Artificial Neural Network (ANN), they displayed a classification performance of 75.9\%. Studies in the other category use EEG records for the diagnosis of migraine. Akben et al. [7] performed feature extraction on the EEG records of migraine patients and HC subjects by applying the AR Burg method. Classifying the features, they obtained with the Support Vector Machine (SVM) classifier, they exhibited 85\% classification success. In another study, Subasi et al. [8] applied the Discrete Wavelet Transform (DWT) method for feature extraction and showed a classification performance of $85.95 \%$ by classifying the results with the Random Forest learning algorithm. Akben et al. [9] in another study, they obtained $83.3 \%$ classification success by classifying the features they obtained by applying AR Burg method with an ANN classifier.

There is no classification study in the literature using the same data set. Therefore, we cannot provide a comparative analysis of the proposed method with a study using the existing data set. The TQWT method is widely used in the literature as a method that provides more information in time frequency analysis of signals. In this respect, we present a study that allows successful differentiation of TQWT-based migraine patients and $\mathrm{HC}$ subjects.

Table 4

A comparative analysis report with relevant literature studies

\begin{tabular}{|c|c|c|c|c|}
\hline & $\begin{array}{l}\text { Feature } \\
\text { Selection/Extraction }\end{array}$ & Classifier & $\begin{array}{l}\text { Classification } \\
\text { Accuracy }\end{array}$ & Data Set \\
\hline Yin ve ark. [4] & ICA & KNN & $90 \%$ & Clinic Review \\
\hline $\begin{array}{l}\text { Krawczyk ve ark. } \\
\text { [5] }\end{array}$ & ReliefF Greedy & Random Forest & $81 \%$ & Clinic Review \\
\hline Akben ve ark. [6] & AR Burg & SVM & $85 \%$ & $\begin{array}{l}\text { EEG ( } 30 \text { patients, } 30 \\
\text { subjects) }\end{array}$ \\
\hline Subasi ve ark. [7] & DWT & Random Forest & $85.95 \%$ & $\begin{array}{l}\text { EEG(15 patients, } 15 \\
\text { subjects) }\end{array}$ \\
\hline Akben ve ark. [8] & AR Burg & ANN & $83.3 \%$ & $\begin{array}{l}\text { EEG ( } 15 \text { patients, } 15 \\
\text { subjects) }\end{array}$ \\
\hline $\begin{array}{l}\text { Jackowski ve ark. } \\
\text { [25] }\end{array}$ & $\begin{array}{l}\text { Backward Feature } \\
\text { Elimination }\end{array}$ & LADTree|NB|NBTree|RBFNetwork & $75.9 \%$ & Clinic Review \\
\hline Our Study & $\begin{array}{l}\text { TQWT and statistical } \\
\text { features }\end{array}$ & Rotation Forest & $89.6 \%$ & $\begin{array}{l}\text { EEG ( } 18 \text { migraine, } 21 \\
\text { subjects) }\end{array}$ \\
\hline
\end{tabular}

\section{Limitations of Study}

There are some shortcomings in this study that can be compensated in future studies. Since there is no specific standard for the TQWT parameters ( $Q$, $r$ and $j$ parameters) that affect the performance of the proposed method, these parameters were determined experimentally in our study. The number of sub bands obtained depends on these parameters. Apart from this, we see the use of default parameters as another deficiency when testing classification performances with different ensemble learning algorithms. In our future studies, we plan to develop approaches in which the necessary parameters for TQWT and ensemble learning algorithms can be selected automatically to differentiate migraine patients and $\mathrm{HC}$ subjects.

\section{Conclusion}

This study introduces a TQWT-based method for the first time to classify migraine patients and their HC subjects. In signal analysis, the discovery of important information over a period of time requires the stability of the signal. Therefore, in order to analyze the oscillatory structure of the EEG signal, the signal is divided into sub bands with the TQWT method. After that, the features are obtained as a result of statistical calculations. The distinctiveness of these features is tested with the KW test. Afterwards, these features obtained for each sub band were tested with different ensemble learning classifiers. The experimental results show that the highest classification performance in the diagnosis of migraine disease was obtained as $89.6 \%$ with the Rotation Forest classifier with the features belonging to Sub band 2 . The

Page $7 / 11$ 
results show that Sub band 2 has the capacity to produce a higher performance in differentiating migraine patients and $\mathrm{HC}$ subjects compared to other sub bands. The proposed method offers a new, robust and consistent approach to automatically diagnose migraine from EEG signals. It is thought that the study we are presenting can support the expert opinion. In future studies, it is planned to achieve higher classification performance by using the state of the art methods for the diagnosis of migraine. It is thought that the proposed method will exhibit high performance by applying to different diseases or different biomedical signals.

\section{Declarations}

\section{Availability of data and material}

The data used in this study are taken from the publicly available data set. Data set is available at " https://kilthub.cmu.edu/articles/dataset/Ultra_highdensity_EEG_recording_of_interictal_migraine_and_controls_sensory_and_rest/12636731" [19].

Conflict of interest The authors declare that they have no conflict of interest.

\section{Compliance with Ethical Standards:}

Ethical approval: This article does not contain any studies with human participants or animals performed by any of the authors.

\section{References}

1. M.R. Foundation, “About Migraine. (Migraine Research Foundation) Retrieved," 2021. [Online]. Available: https://migraineresearchfoundation.org/about-migraine/.

2. T.M. Trust, "Facts and Figures," 2021. [Online]. Available: https://www.migrainetrust.org/about-migraine/migraine-what-is-it/factsfigures/.

3. T. M. Trust, "Diagnosis." [Online]. Available: https://www.migrainetrust.org/living-with-migraine/seeking-medicaladvice/diagnosis/\#: :text=There is no actual test,or other symptoms is taken.

4. H. R. Al Ghayab, Y. Li, S. Siuly, and S. Abdulla, "A feature extraction technique based on tunable Q-factor wavelet transform for brain signal classification,” J. Neurosci. Methods, vol. 312, pp. 43-52, 2019. https://doi.org/10.1016/j.jneumeth.2018.11.014

5. Z. Yin, Z. Dong, X. Lu, S. Yu, X. Chen, and H. Duan, "A clinical decision support system for the diagnosis of probable migraine and probable tension-type headache based on case-based reasoning," J. Headache Pain, vol. 16, no. 1, pp. 1-9, 2015. https://doi.org/10.1186/s10194-015-0512-x

6. B. Krawczyk, D. Simić, S. Simić, and M. Woźniak, "Automatic diagnosis of primary headaches by machine learning methods," Cent. Eur. J. Med., vol. 8, no. 2, pp. 157-165, 2013.

7. S. B. Akben, D. Tuncel, and A. Alkan, “Classification of multi-channel EEG signals for migraine detection.," 2016.

8. A. Subasi, A. Ahmed, E. Aličković, and A. R. Hassan, "Effect of photic stimulation for migraine detection using random forest and discrete wavelet transform," Biomed. Signal Process. Control, vol. 49, pp. 231-239, 2019. https://doi.org/10.1016/j.bspc.2018.12.011

9. S. B. Akben, A. Subasi, and D. Tuncel, "Analysis of repetitive flash stimulation frequencies and record periods to detect migraine using artificial neural network," J. Med. Syst., vol. 36, no. 2, pp. 925-931, 2012. https://doi.org/10.1007/s10916-010-9556-2

10. J. Liu, C. Zhang, Y. Zhu, T. Ristaniemi, T. Parviainen, and F. Cong, "Automated detection and localization system of myocardial infarction in single-beat ECG using Dual-Q TQWT and wavelet packet tensor decomposition," Comput. Methods Programs Biomed., vol. 184, p. 105120, 2020. https://doi.org/10.1016/j.cmpb.2019.105120

11. W. Zeng, J. Yuan, C. Yuan, Q. Wang, F. Liu, and Y. Wang, "A novel technique for the detection of myocardial dysfunction using ECG signals based on hybrid signal processing and neural networks," Soft Comput., vol. 25, no. 6, pp. 4571-4595, 2021. https://doi.org/10.1007/s00500-020-05465-8

12. M. Murugappan, W. Alshuaib, A. K. Bourisly, S. K. Khare, S. Sruthi, and V. Bajaj, “Tunable Q wavelet transform based emotion classification in Parkinson's disease using Electroencephalography," PLoS One, vol. 15, no. 11, p. e0242014, 2020. https://doi.org/10.1371/journal.pone.0242014

13. S. Patidar, R. B. Pachori, A. Upadhyay, and U. R. Acharya, "An integrated alcoholic index using tunable-Q wavelet transform based features extracted from EEG signals for diagnosis of alcoholism," Appl. Soft Comput., vol. 50, pp. 71-78, 2017.

https://doi.org/10.1016/j.asoc.2016.11.002 
14. S. Patidar and T. Panigrahi, "Detection of epileptic seizure using Kraskov entropy applied on tunable-Q wavelet transform of EEG signals," Biomed. Signal Process. Control, vol. 34, pp. 74-80, 2017. https://doi.org/10.1016/j.bspc.2017.01.001

15. S. Patidar, R. B. Pachori, and U. R. Acharya, "Automated diagnosis of coronary artery disease using tunable-Q wavelet transform applied on heart rate signals," Knowledge-Based Syst., vol. 82, pp. 1-10, 2015. https://doi.org/10.1016/j.knosys.2015.02.011

16. A. R. Hassan, S. Siuly, and Y. Zhang, "Epileptic seizure detection in EEG signals using tunable-Q factor wavelet transform and bootstrap aggregating," Comput. Methods Programs Biomed., vol. 137, pp. 247-259, 2016. https://doi.org/10.1016/j.cmpb.2016.09.008

17. S. Taran and V. Bajaj, "Motor imagery tasks-based EEG signals classification using tunable-Q wavelet transform," Neural Comput. Appl., vol. 31, no. 11, pp. 6925-6932, 2019. https://doi.org/10.1007/s00521-018-3531-0

18. V. Bajaj, S. Taran, S. K. Khare, and A. Sengur, "Feature extraction method for classification of alertness and drowsiness states EEG signals," Appl. Acoust., vol. 163, p. 107224, 2020. https://doi.org/10.1016/j.apacoust.2020.107224

19. M. Chaman Zar, Alireza, Haigh, Sarah, Grover, Pulkit, Behrmann, "Ultra high-density EEG recording of interictal migraine and controls: sensory and rest. Carnegie Mellon University. Dataset.," 2020. https://doi.org/10.1184/R1/12636731

20. B. R. Bakshi, “Multiscale PCA with application to multivariate statistical process monitoring," AIChE J., vol. 44, no. 7, pp. 1596-1610, 1998. https://doi.org/10.1002/aic.690440712

21. I. W. Selesnick, “Wavelet transform with tunable Q-factor,” IEEE Trans. signal Process., vol. 59, no. 8, pp. 3560-3575, 2011. doi: 10.1109/TSP.2011.2143711

22. S. Patidar and R. B. Pachori, "Classification of cardiac sound signals using constrained tunable-Q wavelet transform," Expert Syst. Appl., vol. 41, no. 16, pp. 7161-7170, 2014. https://doi.org/10.1016/j.eswa.2014.05.052

23. S. K. Khare and V. Bajaj, "Constrained based tunable Q wavelet transform for efficient decomposition of EEG signals," Appl. Acoust., vol. 163, p. 107234, 2020. https://doi.org/10.1016/j.apacoust.2020.107234

24. S. Narkhede, “Understanding AUC - ROC Curve,” 2021. [Online]. Available: https://towardsdatascience.com/understanding-auc-roc-curve$68 \mathrm{~b} 2303 \mathrm{cc} 9 \mathrm{c} 5$.

25. K. Jackowski, D. Jankowski, D. Simić, and S. Simić, "Migraine diagnosis support system based on classifier ensemble," in International Conference on ICT Innovations, 2014, pp. 329-339.

\section{Figures}

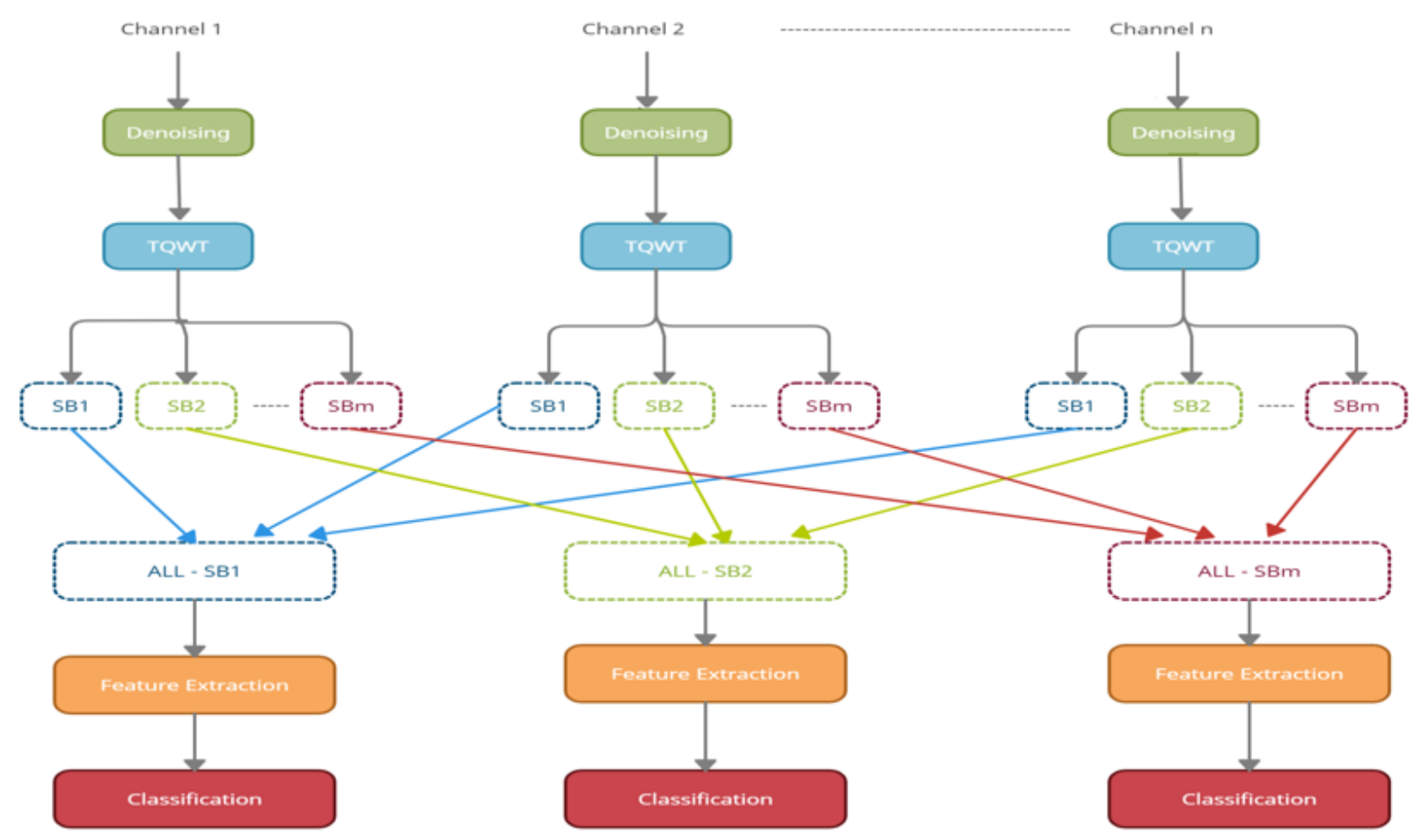

Figure 1 


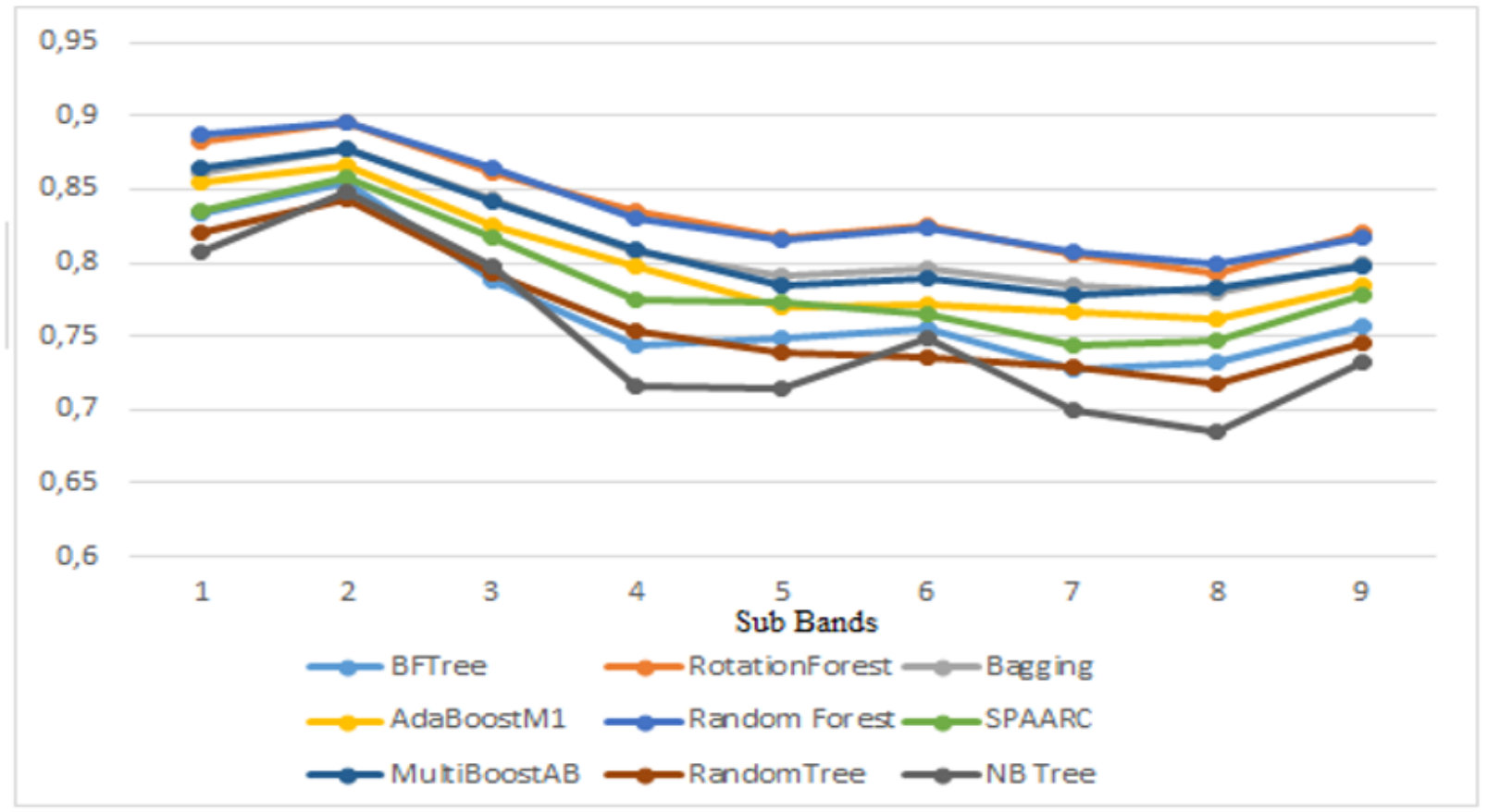

\section{Figure 2}

Classification performances of sub bands obtained with different ensemble classifiers

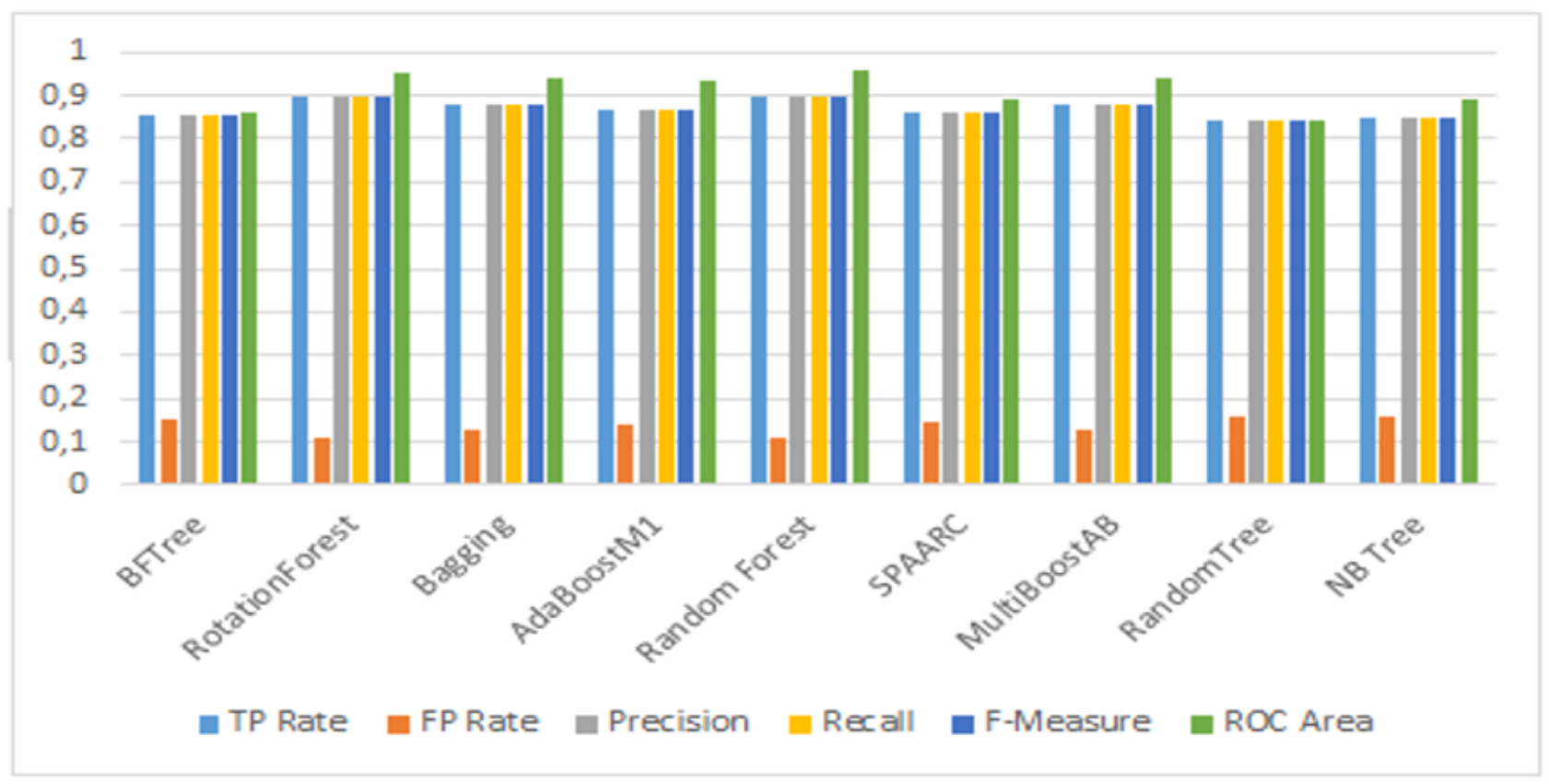

\section{Figure 3}

Evaluation metrics obtained by classifying Sub band 2 with different ensemble classifiers 


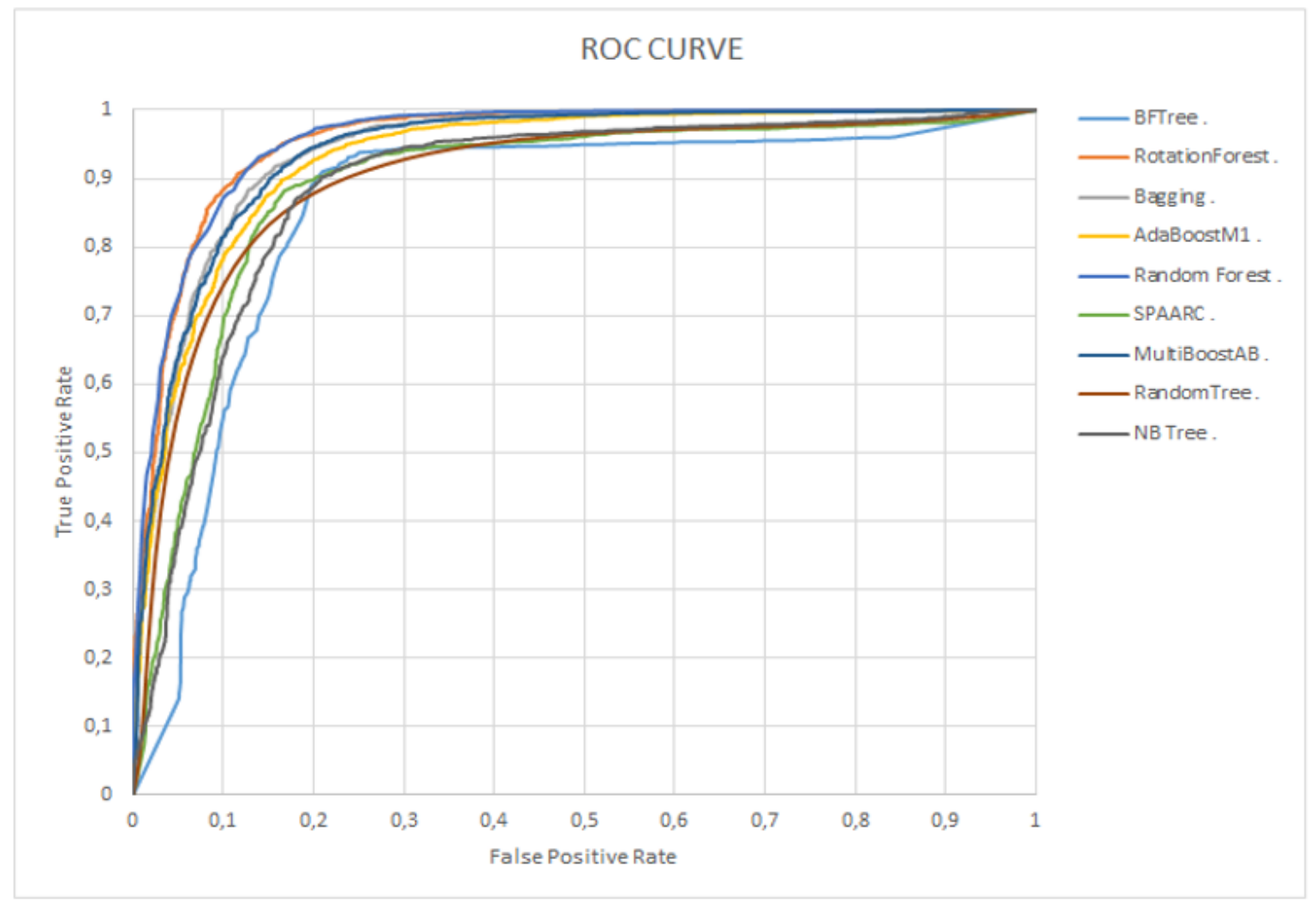

\section{Figure 4}

ROC curve obtained by classifying Sub band 2 with different ensemble learning algorithms.

Predicted

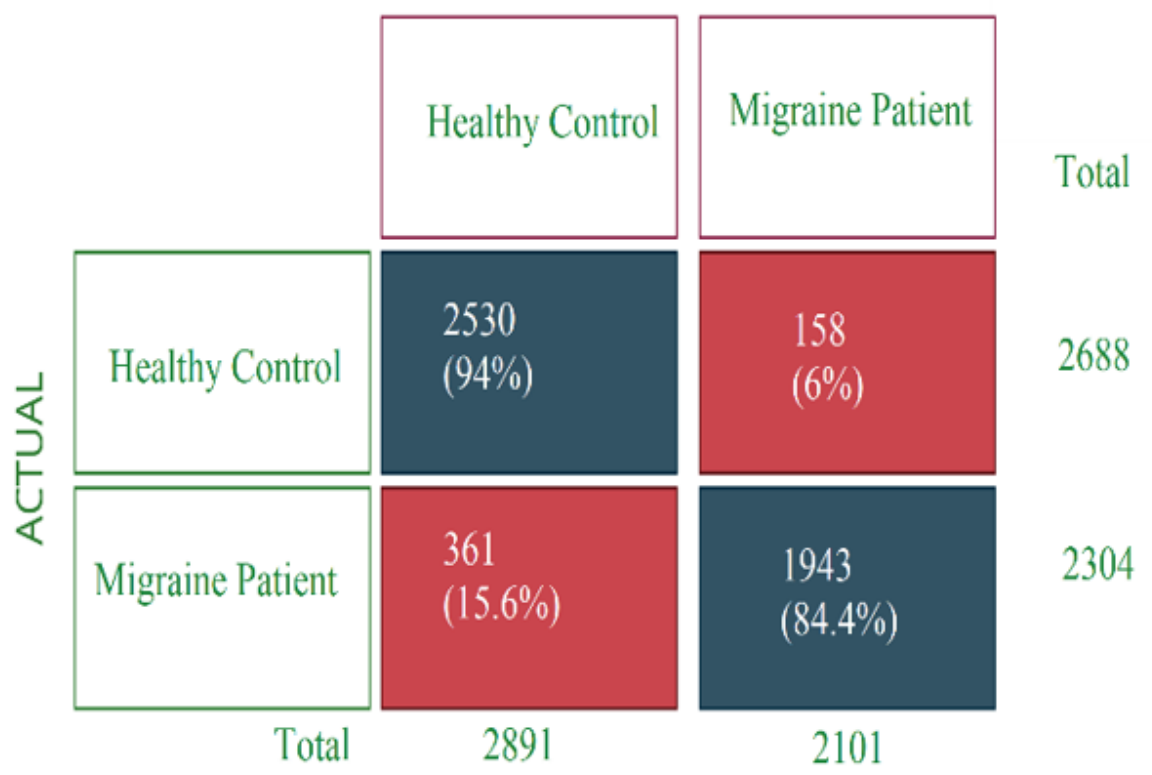

\section{Figure 5}

Confusion Matrix obtained by classifying Sub band 2 with Rotation Forest algorithm. 